\title{
Capacity Enhancement of WDM-TDM Next Generation Bidirectional Passive Optical Networks using DPSK Technique
}

\author{
Baljeet Kaur and Pooja Verma \\ ECE Dept., GNDEC, Ludhiana, Punjab, India
}

\begin{abstract}
Passive Optical Network is designed such that it meets the requirements of NG-PON. According to FSAN, the Time and Wave Length Division Multiplexing (TWDM) is chosen as the best solution to implement NG-PON. EDFA amplifier is used in the system to compensate the effects of degraded signal in both the directions upstream/ Downstream (US/DS). It is observed that system can transmit over $40 \mathrm{Km}$ SMF with acceptable BER. NGPONs also remarkably improves increase in the users to 2400 downstream and 512 upstream. It is observed that with increase of link length the value of $Q$-factor decreases and there is increase in the BER. Capacity enhancement, high data rate and large bandwidth can be achieved by using advanced modulation formats between efficient techniques.
\end{abstract}

Keywords-NG-PON; access networks; WDM; TDM; SOA; DPSK; WDM-PON; Intensity modulation; NRZ; CS-RZ; DRZ

\section{INTRODUCTION}

With the advancement in the communication systems, there is a need for large bandwidth to send more data at higher speed. Optical communication technology gives the solution for higher bandwidth. By developing the optical networks, larger transmission capacity at longer transmission distance can be achieved. Passive Optical Network (PON) is one of the most promising optical access network architectures in term of cost effectiveness [1]. A typical WDM-PON system based on wavelength splitting uses a passive wavelength (de)multiplexer, e.g., arrayed waveguide grating (AWG), in the remote node. Signals are coded on various wavelength channels, and then routed to different ONUs by the (de)multiplexer. The use of a (de)multiplexer avoids the large insertion loss introduced by optical splitter, which greatly improves the power budget of the whole system [3]. This approach also creates a point-to point (P2P) link where a dedicated wavelength channel is reserved between the OLT and each ONU [4]. Moreover, since each ONU only receives its own signals, this point-to-point logical architecture brings in much more privacy and higher security than the TDM-PON technology. Another type of WDM-PON which is referred to as power-splitting approach still uses optical splitter at the remote node, where the data signals with different wavelengths are broadcasted to each ONU.

Then, the optical filters positioned right before the transceivers at the ONUs to select and transmit only one wavelength channel and block all others. This kind of WDMPON can be upgraded easily from the existing TDM-PON architecture without any change in ODN, but it cannot mitigate the problems of low security and high power loss. Time division multiplexing (TDM) can be incorporated together with WDM technology, which is referred as hybrid WDM/TDM technique, to reduce the cost and share the increased bandwidth among subscribers. The cost is shared among all subscribers using TDM splitters for each optical channel. Figure 1 depicts a hybrid WDM/TDM architecture used in later sections of this paper. This basic hybrid network architecture consists of an optical line terminal (OLT), a feeder line, a bidirectional optical amplifier, remote node (RN), access lines including TDM trees, and ONUs.

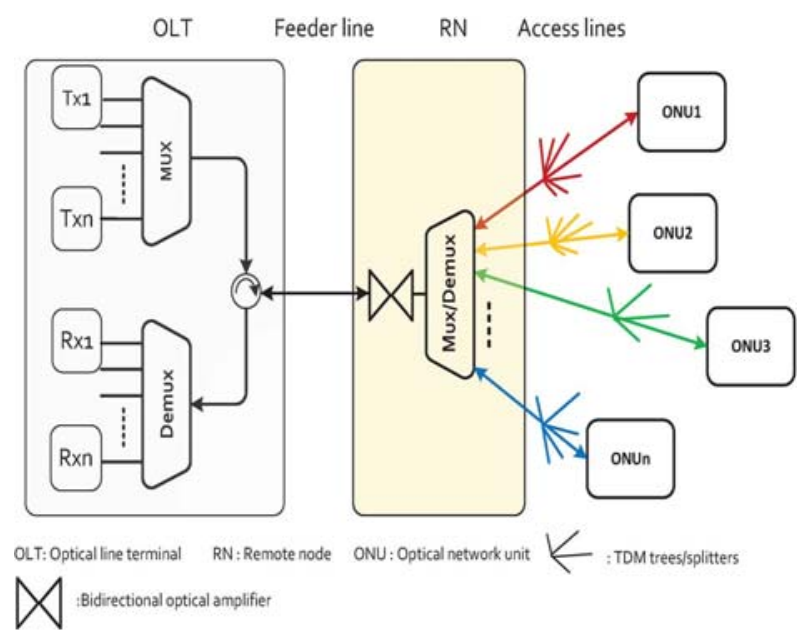

FIGURE I. HYBRID WDM/TDM PON ARCHITECTURE

Different configurations can be used at receiver/transmitter of ONUs such as erbium doped fiber amplifiers (EDFA) depending on the employed technology. The EDFA amplifier is preferred over SOA because in SOA if we increase the number of channels its performance degrades due to increasing gain saturation problems. If we increase the dispersion and number of channels then EDFA provides better results than SOA for higher wavelengths. The optical amplification can be inserted in hybrid WDM/TDM NG-PONs at RN (as in Figure 1) [5]. This paper is based on previous work [8], [9] on efficient SOA-based amplification techniques in NG-PONs. The proposed extension scheme is not only limited to the hybrid WDM/TDM architecture in Figure 1, but also different configurations such as hybrid TWDM NGPON2 [10] can utilize this technique. However, the focus of the paper is to show the significance of the proposed 
amplification configuration considering the coexistence of the current and the future PON technologies.

\section{SYSTEM ARCHITECTURE: EVALUATION OF 60 GBPS WDM-PON SUPPORTING 2400/512 USERS US/DS}

The architecture can support different FTTx (Fiber to the $\mathrm{x}$ ) applications where $\mathrm{x}$ can be home $(\mathrm{H})$, curb (C), building (B), and cell $(\mathrm{C})$. Figure 2 shows the network architecture with the proposed amplification technique. The proposed system consists of 8 transmitter CW lasers from $1550 \mathrm{~nm}$ to $1556.4 \mathrm{~nm}$ wavelengths using RZ-DPSK modulation format. PRBS is used to generate the random data. EDFA amplifier upstream is used to mitigate the effects of attenuation and work as a booster in the setup. A 1x8 power splitter is used to split the signal into 8 equal powers. Two AWG (arrayed waveguide grating) are used each with $80 \mathrm{GHz}$ bandwidth to channelize the different frequency signals to different output ports in downstream as well as in upstream. Circulators are used to provide flexibility and rotation of signals to other ports. B-directional SMF-28 is used for the transmission along with the DCF for pulse width reduction in WDM passive optical network.

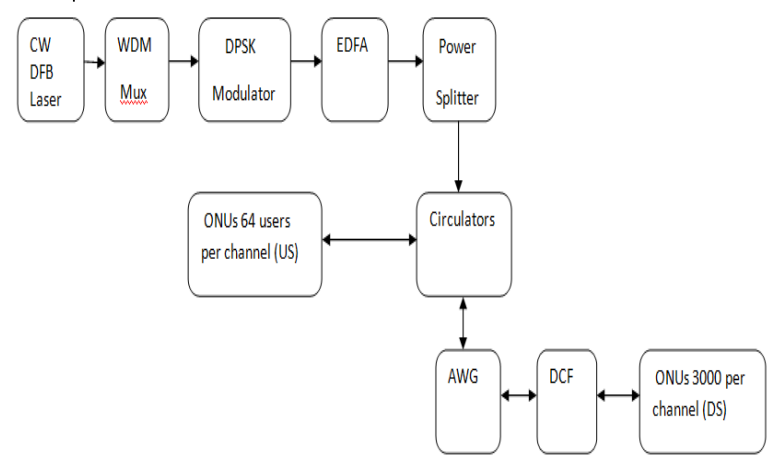

FIGURE II. ARCHITECTURE OF THE PROPOSED SYSTEM

The optical receivers comprises of a PIN photo detector followed by a low pass Bessel filter used for 2400 users each. PIN is used because to detect the optical signal and Bessel filter is used to remove the noises. A 3R regenerator is used for resampling, re-shaping and re-amplification of the signal. Figure 2 shows the architecture of the proposed system. In the current work DPSK modulation technique is performed. DPSK is a constraint type of digital modulation in which the binary input information is contained between two successive signalling elements differences instead of absolute phase. In the proposed work Return to Zero Differential Phase Shift Keying (RZ-DPSK) transmitter system is used which consists of PRBS generator, two NRZ pulse generator, two Lithium Niobate Mach-Zehnder (LiNb MZ) modulator and sine wave generator. For every ' 0 ' phase shift is $180^{\circ}$ and for every ' 1 ' phase shift remain unchanged. Further this modulated signal is transferred both at upstream and downstream to provide bit rates to the users respectively. In this eight channels are used both at upstream and downstream direction.

In downstream and upstream, each channel comprises of 300 users 64 users respectively. Figure 3 represents subsystems for both (a) downstream and (b) upstream frequency. In downstream, power splitter 1:300 is used i.e. power is splitted among 300 users and the power is further transmitted to the DPSK receiver. DPSK removes the need for a coherent reference signal at receiver by combining operationsdifferential encoding and phase shift keying at transmitter.

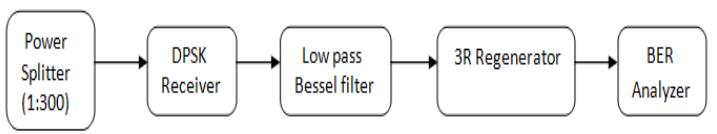

(a)

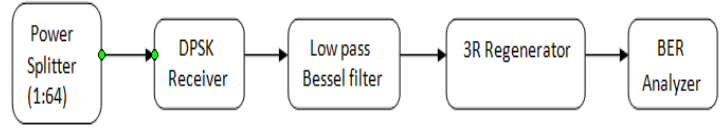

(b)

FIGURE III. SUBSYSTEM FOREACH CHANNEL (A) DOWNSTREAM, AND (B) UPSTREAM

In upstream, power splitter 1:64 is used i.e. power is splitted among 64 users and same process is performed.

\section{A. Downstream Configuration}

The experimental setup is for $8 \times 20$ Gbit/s return-tozero(RZ)-DPSK Bidirectional WDM/TDM-PON as shown in Figure 3(a). Distance of the link length is varied to study the effects of distance on 2400 user PON. The central office is emulated by a Pulse Pattern Generator (PPG) with Clock (Clk) at $10 \mathrm{GHz}$, the NRZ to RZ-DPSK converter, eight distributed feedback (DFB) lasers operating at the following wavelengths: 1550, 1550.8, 1551.6, 1552.4, 1553.2, 1554, 1554.8 and 1555.2; a dual drive electrical amplifier followed by a MachZehnder modulator (MZM), two AWG, and an EDFA. The MZM is loaded from both sides to operate at push-pull in order to increase the extinction ratio. The wavelengths are demultiplexed and multiplexed again to de-synchronize the data patterns on each wavelength. EDFA is used after mux to compensate the mux and demux losses.

\section{B. Upstream Configuration}

The experimental setup is for $8 \times 20 \mathrm{Gbit} / \mathrm{s}$ return-to-zero (RZ)-DPSK Dense WDM/TDM-PON as shown in Figure 3(b). The experimental setup uses cost effective chirped managed lasers as ONUs [14]. Distance of the link length is varied for studying the various effects of distance on 512 users. The driving electrical signal is encoded in inverse return-to-zero (IRZ) format. By direct modulation, a corresponding frequency shift is generated due to the adiabatic chirp of the laser. As a consequence, in order to obtain a phase shift with a $50 \%$-duty-cycle IRZ signal, a maximum frequency shift of about $10 \mathrm{GHz}$ is required. The wavelengths are at 1550 , 1549.2, 1548.4, 1547.6, 1546.8, 1546, 1545.2 and 1544.4, respectively.

\section{EXPERIMENTAL INVESTIGATIONS}

The measurements are performed by having the optical power budget in mind. The feeder budget is defined as the optical power loss from CO to the RN. The performance of WDM-PON system is being observed with a channel spacing of $0.8 \mathrm{~nm}$ with the capacity of $20 \mathrm{Gbits} / \mathrm{s}$ per channel both 
US/DS for obtaining Q-factor. Figure 4 represents the performance analysis of different 8 WDM channels for (a) DS and (b) US supporting 2400 and 512 users respectively. It shows the variations in the Q-factor with respect to transmission distance for 8 WDM channels both for DS/US.

\section{A. Downstream}

The graph shown in figure 4(a) represents variation in Qfactor due to increase in distance for downstream from $10 \mathrm{~km}$ to $50 \mathrm{~km}$.. In the system eight channels/wavelengths have been used for both upstream and downstream. The channel spacing between each channel is of $0.8 \mathrm{~nm} .1350 \mathrm{~nm}$ and $1550 \mathrm{~nm}$ are the favourable wavelength used for demonstration and is considered best for the system performance in WDM PON. In this, the wavelength used is $1550 \mathrm{~nm}$. The wavelengths along eight channels are described in section 2.1. The graph shows that with increasing distance the value of $\mathrm{Q}$ - factor of the system decreases for the distance The maximum Q-factor achieved in downstream is $35 \mathrm{~dB}$.

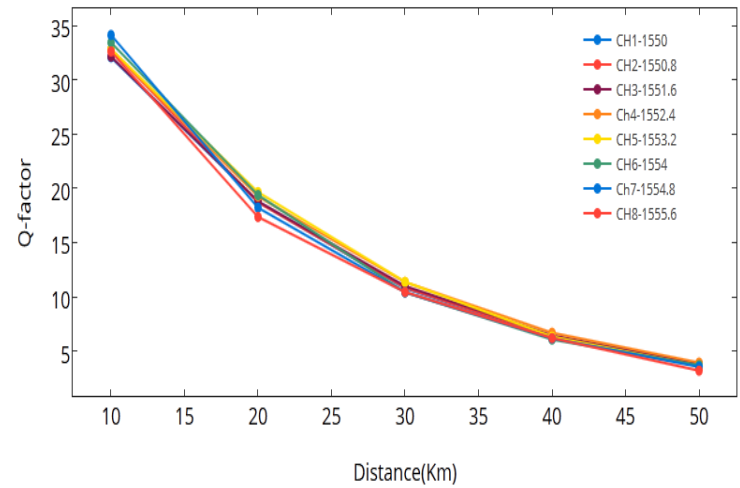

(a)

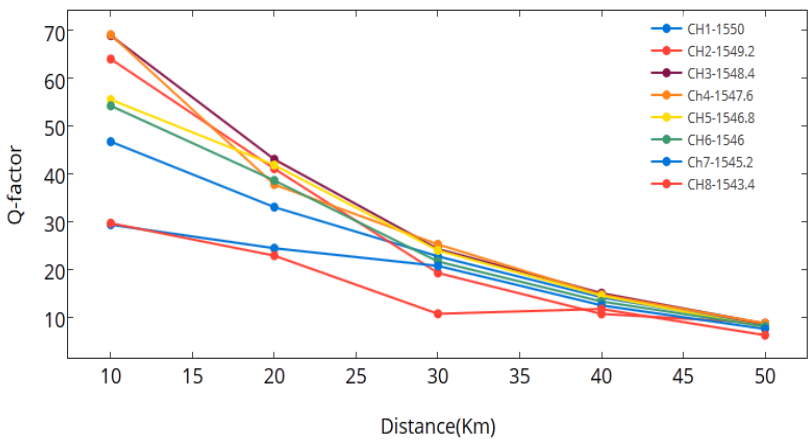

(b)

FIGURE IV. Q FACTOR VS TRANSMISSION DISTANCE FOR 8 WDM CHANNELS (A) DOWNSTREAM (B) UPSTREAM

\section{B. Upstream}

The graph shown in Figure 4(b) represents variation in Qfactor due to increase in distance for upstream. The proposed system operates for $8 \times 8$ channels for both upstream and downstream.In upstream wavelength of the channels gets decreased by the value of channel spacing with respect to the previous wavelength used.
TABLE I. Q-FACTOR VS DISTANCE FOR UPSTREAM AND DOWNSTREAM

\begin{tabular}{|c|c|c|c|c|}
\hline \multirow{2}{*}{$\begin{array}{c}\text { Distance } \\
(\mathbf{K m})\end{array}$} & \multicolumn{2}{|c|}{$\begin{array}{c}\text { 2400 Users } \\
\text { Downstream }\end{array}$} & \multicolumn{2}{c|}{$\mathbf{2 1 2}$ Users Upstream } \\
\cline { 2 - 5 } & $\begin{array}{c}\text { Q- } \\
\text { factor }\end{array}$ & BER & $\begin{array}{c}\text { Q- } \\
\text { Factor }\end{array}$ & BER \\
\hline 10 & 32.07 & $4.21 * 10^{-226}$ & 46.7 & 0 \\
\hline 20 & 18.77 & $5.63^{*} 10^{-79}$ & 32.99 & $4.98 * 10^{-239}$ \\
\hline 30 & 10.79 & $1.85 * 10^{-27}$ & 22.69 & $2.43^{*} 10^{-114}$ \\
\hline 40 & 6.25 & $1.94 * 10^{-10}$ & 14.1 & $1.87 * 10^{-45}$ \\
\hline 50 & 3.66 & 0.000125 & 8.33 & $3.9 * 10^{-17}$ \\
\hline
\end{tabular}

The graph shows that with increasing distance the Q-factor of the system decreases. Q-factor is different for all wavelengths at the distance varying from $10 \mathrm{~km}$ to $50 \mathrm{~km}$. The variations in the Q-factor, BER and distance are shown in table 1 for US and DS frequencies. It represents the highest observed distance and Q-factor for 2400 users in downstream and 512 users in upstream.

\section{CONCLUSION}

WDM-PON is the point to multipoint mechanism to achieve the higher data rats and longer transmission. It is observed that the system supports 2400/512 users and covers $48 \mathrm{Km}$ of distance with acceptable BER of $6.25 * 10^{-10}$. Use of DCF enhance the system performance and system use 1:300 user and 1:64 splitting ratio for each channel for DS and US. Also comparison of different WDM channels has been done and it is observed that $1548.4 \mathrm{~nm}$ performs best in upstream and $1553.2 \mathrm{~nm}$ in downstream transmission. The bit rate of the system increased to $160 \mathrm{Gbps}$ both at downstream upstream hence enhances the system performance. It provides high definition video quality on various bandwidths which is effective for future video services.

\section{REFERENCES}

[1] P. Piskarskas, A. P. Stabinis, and V. Pyragaite, "Ultrabroad bandwidth of optical parametric amplifiers,” IEEE J. Quantum Electron., vol. 46, no. 7, pp. 1031-1038, Jul. 2010

[2] S. Dahlfort, "Comparison of 10 Gbit/s PON vs WDM-PON," in: ECOC, 2009.

[3] J. S. Vardakas, I. D. Moscholios, M. D. Logothetis, and V. G. Stylianakis, "An analytical approach for dynamic wavelength allocation in WDM-TDMA PONs servicing ON-OFF traffic,” J. Opt. Commun. Network, vol. 3, pp. 347-358, April 2011.

[4] B. Schrenk, G. Valicourt, J. A. Lazaro, R. Brenot, and J. Prat, "Rayleigh scattering tolerant PON assisted by four-wave mixing in SOA based ONUs,” J. Lightwave Technology, vol. 28, pp. 3364-3371, December 2010.

[5] F. Saliou, P. Chanclou, F. Laurent, N. Genay, J. A. Lazaro, F. Bonada, and J. Prat, "BReach extension strategies for passive optical networks," IEEE/OSA J. Opt. Commun. Network., vol. 1, no. 4, pp. C51-C60, Sep. 2009.

[6] N. Genay, T. Soret, P. Chanclou, B. Landousies, L. Guillo, and F. Saliou, "Evaluation of the budget extension of a GPON by EDFA amplification,” presented at the Int. Conf. Transparent Optical Networks, 2007.

[7] D. Nesset and P. Wright, "Raman extended GPON using $1240 \mathrm{~nm}$ semiconductor quantum-dot lasers," presented at the Optical Fiber Communication Conf., OSA Technical Digest (CD) Optical Society of America, 2010. 
[8] A. Emsia, T. Q. Le, D. Briggmann, and F. Kueppers, "WDM-PON upstream budget extension for 4 _ $10 \mathrm{Gbit} / \mathrm{s}$ DPSK directly modulated lasers,” in Proc. IEEE IPC, pp. 34-35, , Sep. 2012. 